\title{
PENYELESAIAN MASALAH KONTROL KUADRATIK LINIER YANG MEMUAT FAKTOR DISKON
}

\author{
MEZI FAUZIATUL HUSNA \\ Program Studi Matematika, \\ Fakultas Matematika dan Ilmu Pengetahuan Alam, Universitas Andalas, \\ Kampus UNAND Limau Manis Padang, Indonesia, \\ mezalg_right@gmail.com
}

\begin{abstract}
The linear quadratic control problem is an optimal control problem which has been used in various fields. In this paper, we will study the solving of linear quadratic control problem that contains a discount factor. By using the change of variables technique, some sufficient condition for the existence of optimal control is determined. Some examples are also presented.
\end{abstract}

Kata Kunci: Linear quadratic control, discount factor, algebra Riccati equation

\section{Pendahuluan}

Masalah kontrol kuadratik merupakan masalah penentuan suatu pengontrol optimal $\mathbf{u}^{*}(t) \in \mathbb{R}^{m}$ yang meminimumkan fungsional

$$
J(\mathbf{u})=\int_{0}^{\infty}\left[\mathbf{x}^{T}(t) Q \mathbf{x}(t)+\mathbf{u}^{T}(t) R \mathbf{u}(t)\right] d t
$$

dan memenuhi sistem dinamik

$$
\dot{\mathbf{x}}(t)=A \mathbf{x}(t)+B \mathbf{u}(t), \mathbf{x}(0)=\mathbf{x}_{0}, t \geq 0,
$$

di mana $\mathbf{x} \in \mathbb{R}^{n}$ menyatakan variabel keadaan, $A \in \mathbb{R}^{n \times n}$ dan $B \in \mathbb{R}^{n \times m}$. Matriks $Q \geq 0$ dan $R>0$ adalah matriks simetri dengan unsur-unsur berupa konstanta riil.

Masalah kontrol kuadratik pertama kali diperkenalkan oleh Bolza [2] dalam tahun 1904. Penyelesaian dari masalah ini adalah

$$
\mathbf{u}^{*}(t)=-R^{-1}(P B)^{T} \mathbf{x}(t),
$$

di mana $P$ adalah solusi definit positif tunggal dari persamaan aljabar Riccati

$$
A^{T} P+P A+Q-P B R^{-1}(P B)^{T}=0
$$

dan $\mathbf{x}(t)$ memenuhi persamaan diferensial

$$
\dot{\mathbf{x}}(t)=\left(A-B R^{-1}(P B)^{T}\right) \mathbf{x}(t), \mathbf{x}(0)=\mathbf{x}_{0} .
$$

Seiring dengan perkembangan dalam berbagai bidang, permasalahan kontrol kuadratik telah mengalami berbagai modifikasi. Salah satunya adalah penyisipan 
faktor diskon $e^{2 \alpha t}, \alpha \in \mathbb{R}$ ke dalam fungsional tujuan sedemikian sehingga $J(\mathbf{u})$ dapat ditulis menjadi

$$
J(\mathbf{u})=\int_{0}^{\infty}\left[\mathbf{x}^{T}(t) Q \mathbf{x}(t)+\mathbf{u}^{T}(t) R \mathbf{u}(t)\right] e^{2 \alpha t} d t,
$$

(untuk penjelasan lebih detail, lihat $[1,3,4]$. Dalam makalah ini akan dikaji masalah kontrol kuadratik yang memuat faktor diskon.

\section{Penyelesaian Masalah Kontrol Kuadratik Linier yang Memuat Faktor Diskon}

Dalam bagian ini akan dijabarkan syarat cukup yang menjamin eksistensi pengontrol optimal $\mathbf{u}^{*}(t)$ yang meminimumkan (1.3) dan memenuhi sistem dinamik (1.2). Untuk tujuan ini, terlebih dahulu dilakukan perubahan variabel. Misalkan

$$
\mathbf{y}=\mathbf{x} e^{\alpha t} \operatorname{dan} \mathbf{v}=\mathbf{u} e^{\alpha t},
$$

maka

$$
\dot{\mathbf{y}}=\alpha I \mathbf{x} e^{\alpha t}+\dot{\mathbf{x}} e^{\alpha t},
$$

atau dapat ditulis

$$
\dot{\mathbf{x}}=\dot{\mathbf{y}} e^{-\alpha t}-\alpha I \mathbf{x} .
$$

Akibatnya, persamaan (1.2) ekivalen dengan

$$
\dot{\mathbf{y}}=(\alpha I+A) \mathbf{y}+B \mathbf{v},
$$

dan

$$
\mathbf{y}(0)=\mathbf{x}_{\mathbf{0}} .
$$

Selain itu,

$$
\mathbf{x}^{T} Q \mathbf{x}+\mathbf{u}^{T} R \mathbf{u}=\left[\mathbf{y}^{T} Q \mathbf{y}+\mathbf{v}^{T} R \mathbf{v}\right] e^{-2 \alpha t},
$$

sehingga

$$
\left[\mathbf{x}^{T} Q \mathbf{x}+\mathbf{u}^{T} R \mathbf{u}\right] e^{2 \alpha t}=\mathbf{y}^{T} Q \mathbf{y}+\mathbf{v}^{T} R \mathbf{v} .
$$

Dengan demikian, permasalahan meminimumkan fungsional tujuan (1.3) dengan kendala (1.2) berubah menjadi permasalahan meminimumkan fungsional

$$
J(\mathbf{v})=\int_{0}^{\infty}\left[\mathbf{y}(t)^{T} Q \mathbf{y}(t)+\mathbf{v}(t)^{T} R \mathbf{v}(t)\right] d t,
$$

dengan kendala

$$
\dot{\mathbf{y}}=(\alpha I+A) \mathbf{y}+B \mathbf{v}, \quad \mathbf{y}(0)=\mathbf{x}_{\mathbf{0}} .
$$

Dengan mengupayakan penulisan fungsional tujuan (2.6) ke dalam bentuk berikut:

$$
J(\mathbf{v})=J_{0}+\int_{0}^{\infty}\left(\mathbf{v}-\mathbf{v}_{0}\right)^{T} R\left(\mathbf{v}-\mathbf{v}_{0}\right) d t
$$


dimana konstanta $J_{0}$ adalah suatu entitas yang bebas dari $\mathbf{v}$, dan $\mathbf{v}_{0}$ adalah suatu kontrol baru yang akan dipilih, maka permasalahan yang muncul dari (2.8) adalah bagaimana bentuk eksplisit dari $J_{0}$ dan $\mathbf{v}_{0}(t)$ ?

Jelas bahwa, jika (2.6) dapat dinyatakan dalam bentuk (2.8) maka minimum $J(\mathbf{v})$ dicapai pada $\mathbf{v}=\mathbf{v}_{0}, t \geq t_{0}$, dengan nilai minimum adalah $J_{0}$. Lema berikut diperlukan untuk mendapatkan kontrol optimal $\mathbf{v}^{*}(t)$.

Lema 2.1. Misalkan $P$ adalah suatu matriks simetri. Jika $\lim _{t \rightarrow \infty} \mathbf{y}(t)=\mathbf{0}$, untuk setiap kontrol $\mathbf{v}(t)$ dengan $t \in[0, \infty)$, maka

$$
\int_{0}^{\infty}\left[\mathbf{y}^{T}\left((A+\alpha I)^{T} P+P(A+\alpha I)\right) \mathbf{y}+2 \mathbf{y}^{T} P B \mathbf{v}\right] d t=-\mathbf{y}^{T}(0) P \mathbf{y}(0) .
$$

Bukti. Misalkan $P$ adalah suatu matriks simetri, maka

$$
\begin{aligned}
\int_{0}^{\infty}\left[\mathbf{y}^{T}\right. & \left.\left((A+\alpha I)^{T} P+P(A+\alpha I)\right) \mathbf{y}+2 \mathbf{y}^{T} P B \mathbf{v}\right] d t \\
& =\int_{0}^{\infty}\left[\left(\mathbf{y}^{T}\left(A^{T}+\alpha I\right)+\mathbf{v}^{T} B^{T}\right) P \mathbf{y}+\mathbf{y}^{T} P((A+\alpha I) \mathbf{y}+B \mathbf{v})\right] d t \\
& =\int_{0}^{\infty}\left(\dot{\mathbf{y}}^{T} P \mathbf{y}+\mathbf{y}^{T} P \dot{\mathbf{y}}\right) d t \\
& =\lim _{t \rightarrow \infty} \int_{0}^{t} \frac{d}{d t}\left(\mathbf{y}^{T} P \mathbf{y}\right) d t \\
& =\lim _{t \rightarrow \infty}\left(\left.\mathbf{y}^{T} P \mathbf{y}\right|_{0} ^{t}\right) \\
& =\lim _{t \rightarrow \infty}\left[\mathbf{y}^{T}(t) P \mathbf{y}(t)\right]-\mathbf{y}^{T}(0) P \mathbf{y}(0) \\
& =-\mathbf{y}^{T}(0) P \mathbf{y}(0) .
\end{aligned}
$$

Berdasarkan Lema 2.1, maka $J(\mathbf{v})$ pada persamaan (2.6) dapat ditulis menjadi

$$
\begin{aligned}
J(\mathbf{v})= & \mathbf{y}^{T}(0) P \mathbf{y}(0)+\int_{0}^{\infty}\left[\mathbf{y}^{T}\left(\left(A^{T}+\alpha I\right) P+P(A+\alpha I)+Q\right) \mathbf{y}+\mathbf{v}^{T} R \mathbf{v}\right. \\
& \left.+2 \mathbf{y}^{T} P B \mathbf{v}\right] d t
\end{aligned}
$$

Selanjutnya, misalkan

$$
\mathbf{v}_{0}=-R^{-1}(P B)^{T} \mathbf{y}
$$

maka

$$
\begin{aligned}
\left(\mathbf{v}-\mathbf{v}_{0}\right)^{T} R\left(\mathbf{v}-\mathbf{v}_{0}\right)= & \left(\mathbf{v}+R^{-1}(P B)^{T} \mathbf{y}\right)^{T} R\left(\mathbf{v}+R^{-1}(P B)^{T} \mathbf{y}\right) \\
= & \mathbf{v}^{T} R \mathbf{v}+\mathbf{v}^{T}(P B)^{T} \mathbf{y}+\mathbf{y}^{T}(P B)\left(R^{-1}\right)^{T} R \mathbf{v} \\
& +\mathbf{y}^{T}(P B)\left(R^{-1}\right)^{T} R R^{-1}(P B)^{T} \mathbf{y} \\
= & \mathbf{v}^{T} R \mathbf{v}+\mathbf{v}^{T}(P B)^{T} \mathbf{y}+\mathbf{y}^{T}(P B)\left(R^{-1}\right)^{T} R^{T} \mathbf{v} \\
& +\mathbf{y}^{T}(P B)\left(R^{-1}\right)^{T} R^{T} R^{-1}(P B)^{T} \mathbf{y} \\
= & \mathbf{v}^{T} R \mathbf{v}+2 \mathbf{y}^{T} P B \mathbf{v}+\mathbf{y}^{T}(P B) R^{-1}(P B)^{T} \mathbf{y}
\end{aligned}
$$

atau dapat ditulis

$$
\mathbf{v}^{T} R \mathbf{v}+2 \mathbf{y}^{T} P B \mathbf{v}=\left(\mathbf{v}-\mathbf{v}_{0}\right)^{T} R\left(\mathbf{v}-\mathbf{v}_{0}\right)-\mathbf{y}^{T}(P B) R^{-1}(P B)^{T} \mathbf{y} .
$$


Dengan menggantikan (2.10) ke dalam (2.9), diperoleh

$$
J(\mathbf{v})=J_{0}+\int_{0}^{\infty}\left(\mathbf{v}-\mathbf{v}_{0}\right)^{T} R\left(\mathbf{v}-\mathbf{v}_{0}\right) d t,
$$

di mana

$$
\begin{aligned}
J_{0}= & \mathbf{y}^{T}(0) P \mathbf{y}(0) \\
& +\int_{0}^{\infty}\left[\mathbf{y}^{T}\left(\left(A^{T}+\alpha I\right) P+P(A+\alpha I)+Q-(P B) R^{-1}(P B)^{T}\right) \mathbf{y}\right] d t .
\end{aligned}
$$

Untuk menjamin agar $J_{0}$ bebas dari v, pilih matriks simetri $P$ sedemikian sehingga

$$
\left(A^{T}+\alpha I\right) P+P(A+\alpha I)-P B R^{-1} B^{T} P+Q=0 .
$$

Jadi, pengontrol optimal untuk sistem yang baru adalah

$$
\mathbf{v}^{*}(t)=\mathbf{v}_{0}(t)=-R^{-1}(P B)^{T} \mathbf{y}(t), t \geq 0,
$$

di mana $P$ memenuhi persamaan (2.11) dan $\mathbf{y}(t)$ memenuhi persamaan diferensial

$$
\dot{\mathbf{y}}(t)=\left(A-B R^{-1}(P B)^{T}\right) \mathbf{y}(t), \quad \mathbf{y}(0)=\mathbf{y}_{0}
$$

Dengan menggunakan hubungan (2.1) maka kontrol optimal untuk permasalahan (1.2) dan (1.3) adalah

$$
\begin{aligned}
\mathbf{u} & =\mathbf{v} e^{-\alpha t} \\
\mathbf{u}^{*} & =-R^{-1}(P B)^{T} \mathbf{y}(t) e^{-\alpha t} \\
& =-R^{-1}(P B)^{T} \mathbf{x} e^{\alpha t} e^{-\alpha t} \\
& =-R^{-1}(P B)^{T} \mathbf{x},
\end{aligned}
$$

dimana $P$ memenuhi persamaan (2.11). Dari hubungan $(2.2)$ dan $(2.7), \mathbf{x}(t)$ memenuhi persamaan diferensial

$$
\begin{aligned}
\alpha I \mathbf{x} e^{\alpha t}+\dot{\mathbf{x}} e^{\alpha t} & =(\alpha I+A) \mathbf{x} e^{\alpha t}+B \mathbf{u} e^{\alpha t} \\
\dot{\mathbf{x}} & =(\alpha I+A) \mathbf{x}+B \mathbf{u}-\alpha I \mathbf{x} \\
& =\left(A-B R^{-1}(P B)^{T}\right) \mathbf{x} .
\end{aligned}
$$

Proses ini telah membuktikan teorema berikut.

Teorema 2.2. Untuk masalah kuadratik linier (1.2) dan (1.3), misalkan terdapat suatu matriks simetri definit positif $P$ yang memenuhi persamaan (2.11) dan semua nilai eigen matriks $A-B R^{-1}(P B)^{T}$ memiliki bagian riil negatif, maka

$$
\mathbf{u}^{*}(t)=K \mathbf{x}(t), t \geq 0
$$

di mana

$$
K=-R^{-1}(P B)^{T},
$$

meminimumkan $J(\mathbf{u})$, dan $J^{*}(\mathbf{u})=\mathbf{x}^{T}(0) P \mathbf{x}(0)$. 
Penyelesaian Masalah Kontrol Kuadratik Linier Yang Memuat Faktor Diskon 69

Contoh berikut mengilustrasikan untuk permasalahan kontrol kuadratik yang memuat faktor diskon.

$$
\begin{aligned}
\min J(u) & =\int_{0}^{\infty}\left(8 x_{1}{ }^{2}-6 x_{1} x_{2}+4 x_{2}{ }^{2}+\frac{1}{2} u^{2}\right) e^{4 t} d t \\
\text { s.t. } \dot{x_{1}} & =x_{2}, \quad \dot{x_{2}}=x_{1}+u, \quad x_{1}(0)=0, \quad x_{2}(0)=1 .
\end{aligned}
$$

Masalah (2.14) dapat ditulis menjadi

$$
\begin{gathered}
\min J=\int_{0}^{\infty}\left[\left(\begin{array}{ll}
x_{1} & x_{2}
\end{array}\right)\left(\begin{array}{cc}
8 & -3 \\
-3 & 4
\end{array}\right)\left(\begin{array}{l}
x_{1} \\
x_{2}
\end{array}\right)+\frac{1}{2} u^{2}\right] e^{4 t} d t, \\
\text { s.t. }\left(\begin{array}{c}
\dot{x_{1}} \\
\dot{x_{2}}
\end{array}\right)=\left(\begin{array}{ll}
0 & 1 \\
1 & 0
\end{array}\right)\left(\begin{array}{l}
x_{1} \\
x_{2}
\end{array}\right)+\left(\begin{array}{l}
0 \\
1
\end{array}\right) u, \\
x_{1}(0)=0 \quad x_{2}(0)=1 .
\end{gathered}
$$

Misalkan

$$
\mathbf{y}=\mathbf{x} e^{2 t} \quad, \quad \mathbf{v}=\mathbf{u} e^{2 t} \quad \text { dan } \quad P=\left(\begin{array}{ll}
a & b \\
b & c
\end{array}\right) .
$$

Maka permasalahan (2.14) menjadi meminimumkan

$$
J(v)=\int_{0}^{\infty}\left[y(t)^{T} Q y(t)+v(t)^{T} R v(t)\right] d t .
$$

dengan kendala

$$
\begin{gathered}
\left(\begin{array}{l}
\dot{y}_{1} \\
\dot{y}_{2}
\end{array}\right)=\left(\begin{array}{ll}
2 & 1 \\
1 & 2
\end{array}\right) \mathbf{y}+\left(\begin{array}{l}
0 \\
1
\end{array}\right) \mathbf{v}, \\
y_{1}(0)=0, \quad y_{2}(0)=1 .
\end{gathered}
$$

Selanjutnya, dari (2.11) diperoleh

$$
\begin{aligned}
& \left(\begin{array}{ll}
a & b \\
b & c
\end{array}\right)\left(\begin{array}{ll}
2 & 1 \\
1 & 2
\end{array}\right)+\left(\begin{array}{ll}
2 & 1 \\
1 & 2
\end{array}\right)\left(\begin{array}{ll}
a & b \\
b & c
\end{array}\right)-\left(\begin{array}{ll}
a & b \\
b & c
\end{array}\right)\left(\begin{array}{l}
0 \\
1
\end{array}\right) 2 \\
& \left(\begin{array}{ll}
0 & 1
\end{array}\right)\left(\begin{array}{ll}
a & b \\
b & c
\end{array}\right)+\left(\begin{array}{cc}
8 & -3 \\
-3 & 4
\end{array}\right)=\left(\begin{array}{ll}
0 & 0 \\
0 & 0
\end{array}\right) \\
& \left(\begin{array}{cc}
-2 b^{2}+2 b+4 a+8 & a+4 b+c-2 b c-3 \\
a+4 b+c-2 b c-3 & -2 c^{2}+4 c+2 b+4
\end{array}\right)=\left(\begin{array}{ll}
0 & 0 \\
0 & 0
\end{array}\right) .
\end{aligned}
$$

Solusi sistem persamaan non linier (2.19) ini adalah $a=76, b=13$, dan $c=5$. Jadi,

$$
P=\left(\begin{array}{cc}
76 & 13 \\
13 & 5
\end{array}\right)
$$

Sehingga

$$
\begin{aligned}
v^{*}(t) & =-R^{-1}(P B)^{T} y(t) \\
& =-2\left\{\left(\begin{array}{cc}
76 & 13 \\
13 & 5
\end{array}\right)\left(\begin{array}{l}
0 \\
1
\end{array}\right)\right\}^{T}\left(\begin{array}{l}
y_{1}(t) \\
y_{2}(t)
\end{array}\right) \\
& =-26 y_{1}(t)-10 y_{2}(t) .
\end{aligned}
$$


70 Mezi Fauziatul Husna

Selain itu, dari (2.17)

$$
\begin{aligned}
& \left(\begin{array}{l}
\dot{y}_{1}(t) \\
\dot{y}_{2}(t)
\end{array}\right)=\left(\begin{array}{ll}
2 & 1 \\
1 & 2
\end{array}\right)\left(\begin{array}{l}
y_{1}(t) \\
y_{2}(t)
\end{array}\right)+\left(\begin{array}{l}
0 \\
1
\end{array}\right)\left(-26 y_{1}(t)-10 y_{2}(t)\right) \\
& =\left(\begin{array}{c}
2 y_{1}(t)+y_{2}(t) \\
-25 y_{1}(t)-8 y_{2}(t)
\end{array}\right) \\
& =\left(\begin{array}{cc}
2 & 1 \\
-25 & -8
\end{array}\right)\left(\begin{array}{l}
y_{1}(t) \\
y_{2}(t)
\end{array}\right) \text {. }
\end{aligned}
$$

Dari (2.20) diperoleh

$$
\begin{aligned}
& \dot{y_{1}}(t)=2 y_{1}(t)+y_{2}(t) \\
& \dot{y_{2}}(t)=-25 y_{1}(t)-8 y_{2}(t),
\end{aligned}
$$

atau dapat ditulis

$$
\begin{aligned}
& (D-2) y_{1}(t)=y_{2}(t) \\
& (D+8) y_{2}(t)=-25 y_{1}(t) .
\end{aligned}
$$

Misalkan $D=\frac{d}{d t}$, maka (2.21) dapat ditulis menjadi

$$
\begin{aligned}
(D+8)(D-2) y_{1}(t) & =(D+8) y_{2}(t) \\
\left(D^{2}+6 D-16\right) y_{1}(t) & =-25 y_{1}(t) \\
\left(D^{2}+6 D+9\right) y_{1}(t) & =0 \\
\frac{d^{2} y_{1}(t)}{d t^{2}}+6 \frac{d y_{1}(t)}{d t}+9 y_{1}(t) & =0 \\
r^{2}+6 r+9 & =0 \\
(r+3)(r+3) & =0 .
\end{aligned}
$$

Karena $r_{1}=r_{2}=-3$, maka

$$
y_{1}(t)=c_{1} e^{-3 t}+c_{2} t e^{-3 t} .
$$

Akibatnya,

$$
\begin{aligned}
y_{2}(t) & =\dot{y}_{1}(t)-2 y_{1}(t) \\
& =-3 c_{1} e^{-3 t}-3 c_{2} t e^{-3 t}+c_{2} e^{-3 t}-2 c_{1} e^{-3 t}-2 c_{2} t e^{-3 t} \\
& =-5 c_{1} e^{-3 t}-5 c_{2} t e^{-3 t}+c_{2} e^{-3 t} \\
& =-5 c_{1} e^{-3 t}+c_{2} e^{-3 t}(1-5 t)
\end{aligned}
$$

Sehingga solusi umum dari sistem (2.20) adalah

$$
\left(\begin{array}{l}
y_{1}(t) \\
y_{2}(t)
\end{array}\right)=c_{1}\left(\begin{array}{c}
e^{-3 t} \\
-5 e^{-3 t}
\end{array}\right)+c_{2}\left(\begin{array}{c}
t e^{-3 t} \\
(1-5 t) e^{-3 t}
\end{array}\right) .
$$

Dari (2.18), diperoleh

$$
\begin{aligned}
c_{1} & =0 \\
-5 c_{1}+c_{2} & =1,
\end{aligned}
$$


mengakibatkan $c_{2}=1$. Jadi

$$
\left(\begin{array}{l}
y_{1}(t) \\
y_{2}(t)
\end{array}\right)=\left(\begin{array}{c}
t e^{-3 t} \\
(1-5 t) e^{-3 t}
\end{array}\right)
$$

dan

$$
v(t)=2 e^{-3 t}(12 t-5) .
$$

Dengan demikian, kontrol optimal adalah

$$
u(t)=2 e^{-5 t}(12 t-5),
$$

dan keadaan optimal adalah

$$
\left(\begin{array}{l}
x_{1}(t) \\
x_{2}(t)
\end{array}\right)=\left(\begin{array}{c}
t e^{-5 t} \\
(1-5 t) e^{-5 t}
\end{array}\right)
$$

dengan nilai minimum dari $J(u)$ adalah 5 .

\section{Kesimpulan}

Pada makalah ini telah ditunjukkan bahwa kontrol optimal untuk masalah

$$
\begin{gathered}
\min _{\mathbf{u} \in \mathbb{R}^{m}} J(\mathbf{u})=\int_{0}^{\infty}\left[\mathbf{x}^{T}(t) Q \mathbf{x}(t)+\mathbf{u}^{T}(t) R \mathbf{u}(t)\right] e^{2 \alpha t} d t . \\
\text { s.t. } \dot{\mathbf{x}}(t)=A \mathbf{x}(t)+B \mathbf{u}(t), \mathbf{x}(0)=\mathbf{x}_{0}, t \in[0, \infty), \alpha \in \mathbb{R},
\end{gathered}
$$

adalah

$$
\mathbf{u}^{*}(t)=K \mathbf{x}(t), t \geq 0
$$

dengan

$$
K=-R^{-1}(P B)^{T} .
$$

\section{Ucapan Terima kasih}

Penulis mengucapkan terima kasih kepada Bapak Muhafzan, Bapak Effendi, Bapak Bukti Ginting, dan Ibu Izzati Rahmi yang telah memberikan masukan dan saran sehingga makalah ini dapat diselesaikan dengan baik.

\section{Daftar Pustaka}

[1] Anderson, B.D.O and J. B. Moore. 1969. Linear System Optimization with Pescribed Degree of Stability. Proc. IEE. 116: 2083 - 2087

[2] Bolza, O. 1904. Lectures on The Calculus of Variations. The Decennial Publications, Chicago

[3] Chiang, A. C. 1992. Element of Dynamic Optimization. Science Typographers Inc, Singapore

[4] Kamien, M.I and N.L Schwartz. 1991. Dynamic Optimization. Elsevier, Netherlands 